\title{
Inverses of Multivariable Polynomial Matrices by Discrete Fourier Transforms
}

\author{
S. Vologiannidis and N. P. Karampetakis \\ Department of Mathematics, Aristotle University of Thessaloniki \\ Thessaloniki 54006, Greece
}

October 30, 2002

\begin{abstract}
Two discrete Fourier transform based algorithms are proposed for the computation of the Moore-Penrose and Drazin inverse of a multivariable polynomial matrix.

AMS Classification : Primary 15A09, 65T50; Secondary 93B40,93B50, $65 \mathrm{~F} 30,65 \mathrm{~F} 05\}$
\end{abstract}

\section{Introduction}

Let $\mathbb{R}$ be the set of real numbers, $\mathbb{R}^{p \times m}$ be the set of $p \times m$ real matrices, $\mathbb{R}\left[z_{1}, z_{2}, \ldots, z_{n}\right]$ (resp. $\mathbb{R}\left(z_{1}, z_{2}, \ldots, z_{n}\right)$ ) denotes the polynomials (resp. rational expressions) with real coefficients in the $n$ indeterminates $z_{1}, z_{2}, \ldots, z_{n}$. The $p \times m$ matrices with elements in $\mathbb{R}\left[z_{1}, z_{2}, \ldots, z_{n}\right]$ (resp. $\mathbb{R}\left(z_{1}, z_{2}, \ldots, z_{n}\right)$ ) are denoted by $\mathbb{R}\left[z_{1}, z_{2}, \ldots, z_{n}\right]^{p \times m}$ (resp. $\left.\mathbb{R}\left(z_{1}, z_{2}, \ldots, z_{n}\right)^{p \times m}\right)$. By $I_{p}$ we denote the identity matrix of order $p$, and by $0_{p, m}$ the $p \times m$ null matrix. By $A\left(z_{1}, z_{2}, \ldots, z_{n}\right)^{D}$ (resp. $\left.A\left(z_{1}, z_{2}, \ldots, z_{n}\right)^{+}\right)$we denote the Drazin and Moore-Penrose inverse of $A\left(z_{1}, z_{2}, \ldots, z_{n}\right)$.

The Moore-Penrose inverse has originally been defined by Penrose [16], while later Decell [3] proposed a Leverrier-Faddeev algorithm for its computation. An extension of this algorithm to the one and two-variable polynomial matrices has been proposed by [10], [12], [13], [14]. A Leverrier-Faddeev algorithm has also been proposed by Grevile [7] for the computation of the Drazin inverse of square constant matrices with extensions to the one-variable polynomial matrices by [11], [18].

The Leverrier algorithms have the advantage that are easily implemented in symbolic programming languages like Mathematica, Maple etc. However, their main disadvantage, is that are not stable if they are implemented in other high level programming languages such as $\mathrm{C}++$, Fortran etc. In order to overcome these difficulties we may use other techniques such as interpolation methods. Schuster and Hippe [17] for example, use interpolation techniques in order to 
find the inverse of a polynomial matrix. However, if we need to increase the speed and robustness of our algorithms we may interested in finding algorithms based on Discrete Fourier Transforms (DFT) or better Fast Fourier Transforms (FFT). The main advantages of the DFT based algorithms are:

1. There are very efficient algorithms available both in software and hardware.

2. Parallel environment (through symmetric multiprocessing or other techniques) greatly benefits their speed.

Actually during the past two decades there has been extensive use of DFT - based algorithms, due to their computational speed and accuracy. Some remarkable examples, but not the only ones of the use of DFT in linear algebra problems, are the calculation of the determinantal polynomial by [15], the computation of the transfer function of generalized n-dimensional systems by [1] and the solutions of polynomial matrix Diophantine equations by [9].

The main reason for the interest in these two specific inverses are due to their applications in inverse systems, solution of AutoRegressive Moving Average representations [8], solution of Diophantine equations which gives rise to numerous applications to the field of control system synthesis (see for example [12] and its references) and in the study of multidimensional filters which find numerous applications in image processing, electrical networks with variable elements etc. Note that in case of square and nonsingular matrices, both inverses coincide with the known inverse of the matrix. Therefore the computation of these special inverses gives rise also to applications where the usual inverse of a matrix is required such as the computation of the transfer function of a matrix $([2],[1])$.

The main purpose of this work is to present a DFT-algorithm for the evaluation of the generalized inverse and the Drazin inverse of a multivariable poly-

nomial matrix. More specifically in section 2 we introduce the n-dimensional discrete Fourier transform, while later in section 3 and 4 we propose two new DFT algorithms for the evaluation of the generalized and Drazin inverse respectively of a polynomial matrix in $n$ indeterminates. The whole theory is illustrated via an illustrative example coming from the field of control system synthesis.

\section{Multidimensional Discrete Fourier Transform}

Consider the finite sequence $X\left(k_{1}, \ldots, k_{n}\right)$ and $\tilde{X}\left(r_{1}, \ldots, r_{n}\right), k_{i}, r_{i}=0,1, \ldots, M_{i}$. In order for the sequence $X\left(k_{1}, \ldots, k_{n}\right)$ and $\tilde{X}\left(r_{1}, \ldots, r_{n}\right)$ to constitute an DFT pair the following relations should hold [5] :

$$
\tilde{X}\left(r_{1}, \ldots, r_{n}\right)=\sum_{k_{1}=0}^{M_{1}} \sum_{k_{2}=0}^{M_{2}} \ldots \sum_{k_{n}=0}^{M_{n}} X\left(k_{1}, \ldots, k_{n}\right) W_{1}^{-k_{1} r_{1}} \ldots W_{1}^{-k_{n} r_{n}}
$$




$$
X\left(k_{1}, \ldots, k_{n}\right)=\frac{1}{R} \sum_{r_{1}=0}^{M_{1}} \sum_{r_{2}=0}^{M_{2}} \ldots \sum_{r_{n}=0}^{M_{n}} \tilde{X}\left(r_{1}, \ldots, r_{n}\right) W_{1}^{k_{1} r_{1}} \ldots W_{1}^{k_{n} r_{n}}
$$

where

$$
\begin{aligned}
W_{i} & =e^{\frac{2 \pi j}{M_{i}+1}} \forall i=1,2,3, \ldots, n \\
R & =\prod_{i=1}^{n}\left(M_{i}+1\right)
\end{aligned}
$$

and $X, \tilde{X}$ are discrete argument matrix-valued functions, with dimensions $p \times m$. The computation of the multidimensional DFT and its inverse can be also accomplished using fast Fourier transform techniques. A very fast free implementation of FFT can be found in [6].

\section{Generalized Inverse of a Multivariable Poly- nomial Matrix}

The generalized inverse of a constant matrix was defined by Penrose in [16].

Definition 1 [16] For every matrix $A \in \mathbb{R}^{p \times m}$, a unique matrix $A^{+} \in \mathbb{R}^{m \times p}$, which is called generalized inverse, exists satisfying

(i) $A A^{+} A=A$

(ii) $A^{+} A A^{+}=A^{+}$

(iii) $\left(A A^{+}\right)^{T}=A A^{+}$

(iv) $\left(A^{+} A\right)^{T}=A^{+} A$

where $A^{T}$ denotes the transpose of $A$. In the special case that the matrix $A$ is square nonsingular matrix, the generalized inverse of $A$ is simply its inverse i.e. $A^{+}=A^{-1}$.

Consider the polynomial matrix with real coefficients in the $n$ indeterminates $z_{1}, z_{2}, \ldots, z_{n}$ (called $n D$ polynomial matrix)

$$
A\left(z_{1}, \ldots, z_{n}\right)=\sum_{k_{1}=0}^{M_{1}} \sum_{k_{2}=0}^{M_{2}} \ldots \sum_{k_{n}=0}^{M_{n}}\left(A_{k_{1} \ldots k_{n}}\right) \times\left(z_{1}^{k_{1}} \ldots z_{n}^{k_{n}}\right) \in \mathbb{R}\left[z_{1}, \ldots, z_{n}\right]^{p \times m}
$$

with $A_{k_{1} \ldots k_{n}} \in \mathbb{R}^{p \times m}$, and $p$ not necessarily equal to $m$. In an analogous way we define the generalized inverse $A\left(z_{1}, \ldots, z_{n}\right)^{+} \in \mathbb{R}\left(z_{1}, \ldots, z_{n}\right)^{m \times p}$ of the polynomial matrix $A\left(z_{1}, \ldots, z_{n}\right) \in \mathbb{R}\left[z_{1}, \ldots, z_{n}\right]^{p \times m}$ defined in (5) as the matrix which satisfies the properties (i)-(iv) of Definition (1). We will denote

$$
\bar{z}=\left(z_{1}, \ldots, z_{n}\right) \in \mathbb{R}^{n}
$$

and with a slight abuse of notation

$$
a(\bar{z}) \equiv a\left(z_{1}, \ldots, z_{n}\right) \in \mathbb{R}\left[z_{1}, \ldots, z_{n}\right]
$$

will denote a $n D$ polynomial. Following the steps of [13] we have 
Theorem 2 Let $A(\bar{z})=A\left(z_{1}, \ldots, z_{n}\right) \in \mathbb{R}\left[z_{1}, \ldots, z_{n}\right]^{p \times m}$ as in (5) and

$$
\begin{aligned}
& a\left(s, z_{1}, \ldots, z_{n}\right)=\operatorname{det}\left[s I_{p}-A(\bar{z}) A(\bar{z})^{T}\right] \\
& \quad=\left(a_{0}(\bar{z}) s^{p}+\cdots+a_{p-1}(\bar{z}) s+a_{p}(\bar{z})\right)
\end{aligned}
$$

$a_{0}(\bar{z})=1$, be the characteristic polynomial of $A(\bar{z}) \times A(\bar{z})^{T}$. Let $k$ such that $a_{p}(\bar{z}) \equiv 0, \ldots, a_{k+1}(\bar{z}) \equiv 0$ while $a_{k}(\bar{z}) \neq 0$, and define $\Lambda:=\left\{(\bar{z}) \in \mathbb{C}^{n}: a_{k}(\bar{z})=\right.$ $0\}$. Then the generalized inverse $A(\bar{z})^{+}$of $A(\bar{z})$ for $\bar{z} \in \mathbb{C}^{n}-\Lambda$ is given by

$$
\begin{aligned}
A(\bar{z})^{+} & =-\frac{1}{a_{k}(\bar{z})} A(\bar{z})^{T} B_{k-1}(\bar{z}) \\
B_{k-1}(\bar{z}) & =a_{0}(\bar{z})\left[A(\bar{z}) A(\bar{z})^{T}\right]^{k-1}+\cdots+a_{k-1}(\bar{z}) I_{p}
\end{aligned}
$$

If $k=0$ is the largest integer such that $a_{k}(\bar{z}) \neq 0$, then $A(\bar{z})^{+}=0$. For those $\bar{z} \in \Lambda$ we can use the same algorithm again.

Proof. The theorem is easily proved using the logic in the proof of the corresponding theorem about constant matrices in [3].

Remark 3 The algorithm described in (2) is efficient using symbolic programming languages. Its main advantages are

1) It consists of simple recursions.

2) No matrix inversion is required.

3)In the case that $p>m$ we can compute the transpose $A(\bar{z})^{T}$ and compute $A(\bar{z})^{+}=\left[A(\bar{z})^{+}\right]^{T}$. The algorithm will be completed faster since it will need $m$ rather than $p$ steps.

In the following we will propose a new algorithm for the calculation of the generalized inverse which combines the above advantages with numerical stability and robustness by using interpolation and discrete Fourier transforms.

Evaluation of the generalized inverse of $A\left(z_{1}, \ldots, z_{n}\right)$

Step 1.

It is easily seen from $(6)$, that the greatest powers of the $(n+1)$ variables in $a\left(s, z_{1}, \ldots, z_{n}\right)$ are

$$
\begin{gathered}
\operatorname{deg}_{s}\left(a\left(s, z_{1}, \ldots, z_{n}\right)\right)=p:=b_{0} \\
\operatorname{deg}_{z_{1}}\left(a\left(s, z_{1}, \ldots, z_{n}\right)\right) \leq 2 p M_{1}:=b_{1} \\
\vdots \\
\operatorname{deg}_{z_{n}}\left(a\left(s, z_{1}, \ldots, z_{n}\right)\right) \leq 2 p M_{n}:=b_{n}
\end{gathered}
$$

Thus, the polynomial $a\left(s, z_{1}, \ldots, z_{n}\right)$ can be written as

$$
a\left(s, z_{1}, \ldots, z_{n}\right)=\sum_{k_{0}=0}^{b_{0}} \sum_{k_{1}=0}^{b_{1}} \ldots \sum_{k_{n}=0}^{b_{n}}\left(a_{k_{0} k_{1} \ldots k_{n}}\right)\left(s^{k_{0}} z_{1}^{k_{1}} \ldots z_{n}^{k_{n}}\right)
$$

and can be numerically computed via interpolation using the following $R$ points

$$
\begin{gathered}
u_{i}\left(r_{j}\right)=W_{i}^{-r_{j}} ; i=0, \ldots, n \text { and } r_{j}=0,1, \ldots, b_{i} \\
W_{i}=e^{\frac{2 \pi j}{b_{i}+1}}
\end{gathered}
$$


where

$$
R=\prod_{i=0}^{n}\left(b_{i}+1\right)
$$

In order, to evaluate the coefficients $a_{k_{0} k_{1} \ldots k_{n}}$ define

$$
\tilde{a}_{r_{0} r_{1} \ldots r_{n}}=\operatorname{det}\left[u_{0}\left(r_{0}\right) I_{p}-A\left(u_{1}\left(r_{1}\right), \ldots, u_{n}\left(r_{n}\right)\right)\left[A\left(u_{1}\left(r_{1}\right), \ldots, u_{n}\left(r_{n}\right)\right)\right]^{T}\right]
$$

From (9), (10), (11) we get

$$
\tilde{a}_{r_{0} r_{1} \ldots r_{n}}=\sum_{l_{0}=0}^{b_{0}} \sum_{l_{1}=0}^{b_{1}} \ldots \sum_{l_{n}=0}^{b_{n}}\left(a_{l_{0} l_{1} \ldots l_{n}}\right)\left(W_{0}^{-r_{0} l_{0}} \ldots W_{n}^{-r_{n} l_{n}}\right)
$$

Notice that $\left[a_{l_{0} l_{1} \ldots l_{n}}\right]$ and $\left[\tilde{a}_{r_{0} r_{1} \ldots r_{n}}\right]$ form a DFT pair and thus using (2) we have

$$
a_{l_{0} l_{1} \ldots l_{n}}=\frac{1}{R} \sum_{r_{0}=0}^{b_{0}} \sum_{r_{1}=0}^{b_{1}} \ldots \sum_{r_{n}=0}^{b_{n}} \tilde{a}_{r_{0} r_{1} \ldots r_{n}} W_{0}^{r_{0} l_{0}} \ldots W_{n}^{r_{n} l_{n}}
$$

where $l_{i}=0, \ldots, b_{i}$.

Step 2. (Evaluate $a_{k}(\bar{z})$ )

Find $k: a_{k+1}(\bar{z})=a_{k+2}(\bar{z})=\cdots=a_{p}(\bar{z})=0$ and $a_{k}(\bar{z}) \neq 0$

Step 3. (Evaluate $C(\bar{z})=A(\bar{z})^{T} B_{k-1}(\bar{z})$ )

The greatest powers of $z_{i}$ in

$$
C(\bar{z})=A(\bar{z})^{T} B_{k-1}(\bar{z})=a_{0}(\bar{z})\left[A(\bar{z}) A(\bar{z})^{T}\right]^{k-1}+\cdots+a_{k-1}(\bar{z}) I_{p}
$$

is

$$
n_{i}=\max \left\{2(k-1) M_{i}+M_{i}, k=1, \ldots, p\right\}=(2 p-1) M_{i}
$$

Using the previous observation $C(\bar{z})$ can be written as

$$
C(\bar{z})=\sum_{l_{1}=0}^{n_{1}} \ldots \sum_{l_{n}=0}^{n_{n}} C_{l_{0} \cdots l_{n}}\left(z_{1}^{l_{1}} \cdots z_{n}^{l_{n}}\right)
$$

We compute $C(\bar{z})$ via interpolation using the following $R$ points

$$
\begin{gathered}
u_{i}\left(r_{j}\right)=W_{i}^{-r_{j}} ; i=1, \ldots, n \text { and } r_{j}=0,1, \ldots, n_{i} \\
W_{i}=e^{\frac{2 \pi j}{n_{i}+1}}
\end{gathered}
$$

where

$$
R=\prod_{i=1}^{n}\left\{(2 p-1) M_{i}+1\right\}
$$

To evaluate the coefficients $C_{l_{0} \cdots l_{n}}$ define

$$
\tilde{C}_{r_{1} \ldots r_{n}}=C\left(u_{1}\left(r_{1}\right), \ldots u_{n}\left(r_{n}\right)\right)
$$


Using (15), (16), (17) becomes

$$
\tilde{C}_{r_{1} \cdots r_{n}}=\sum_{l_{1}=0}^{n_{1}} \ldots \sum_{l_{n}=0}^{n_{n}} C_{l_{0} \cdots l_{n}} W_{1}^{-r_{1} l_{1}} \cdots W_{n}^{-r_{n} l_{n}}
$$

which through (2)

$$
C_{l_{0} \cdots l_{n}}=\frac{1}{R} \sum_{l_{1}=0}^{n_{1}} \ldots \sum_{l_{n}=0}^{n_{n}} \tilde{C}_{r_{1} \cdots r_{n}} W_{1}^{r_{1} l_{1}} \cdots W_{n}^{r_{n} l_{n}}
$$

where $l_{i}=0, \ldots, n_{i}$.

Step 4. (Evaluation of the generalized inverse)

$$
A(\bar{z})^{+}=-\frac{1}{a_{k}(\bar{z})} C(\bar{z})
$$

\section{Drazin Inverse of a Multivariable Polynomial Matrix}

The Drazin inverse of a constant matrix was defined by Drazin in [4].

Definition 4 For every matrix $A \in \mathbb{R}^{m \times m}$, there exists a unique matrix $A^{D} \in$ $\mathbb{R}^{m \times m}$, which is called Drazin inverse, satisfying

(i) $A^{D} A^{k+1}=A^{k}$ for $k=\operatorname{ind}(A)=\min \left(k \in N: \operatorname{rank}\left(A^{k}\right)=\operatorname{rank}\left(A^{k+1}\right)\right)$

(ii) $A^{D} A A^{D}=A^{D}$

(iii) $A A^{D}=A^{D} A$

In the special case that the matrix $A$ is square and nonsingular matrix, the Drazin inverse of $A$ is simply its inverse i.e. $A^{D}=A^{-1}$.

In an analogous way we define the Drazin inverse of polynomial matrix $A\left(z_{1}, \ldots, z_{n}\right) \in \mathbb{R}\left[z_{1}, \ldots, z_{n}\right]^{m \times m}$ defined in (5) as the matrix which satisfies the properties of Definition (4). The following theorem proposes a new algorithm for the computation of the Drazin inverse of a $n D$ polynomial matrix, which generalizes the results in [18].

Theorem 5 Consider a nonregular nD polynomial matrix $A(\bar{z})$. Assume that

$$
\begin{aligned}
& a\left(s, z_{1}, \ldots, z_{n}\right)=\operatorname{det}\left[s I_{m}-A(\bar{z})\right] \\
= & \left(a_{0}(\bar{z}) s^{m}+\cdots+a_{m-1}(\bar{z}) s+a_{m}(\bar{z})\right)
\end{aligned}
$$

where

$$
a_{0}(\bar{z}) \equiv 1, z \in \mathbb{C}
$$

is the characteristic polynomial of $A(\bar{z})$. Also, consider the following sequence of $m \times m$ polynomial matrices

$$
\begin{gathered}
B_{j}(\bar{z})=a_{0}(\bar{z}) A(\bar{z})^{j}+\cdots a_{j-1}(\bar{z}) A(\bar{z})+a_{j}(\bar{z}) I_{m}, \\
a_{0}(\bar{z})=1, j=0, \ldots, m
\end{gathered}
$$


Let

$$
a_{m}(\bar{z}) \equiv 0, \ldots, a_{t+1}(\bar{z}) \equiv 0, a_{t}(\bar{z}) \neq 0 .
$$

Define the following set:

$$
\Lambda=\left\{\bar{z}_{i} \in \mathbb{C}^{n}: a_{t}\left(\bar{z}_{i}\right)=0\right\}
$$

Also, assume that

$$
B_{m}(\bar{z}), \ldots, B_{r}(\bar{z})=0, B_{r-1}(\bar{z}) \neq 0
$$

and $k=r-t$. In the case $\bar{z} \in \mathbb{C}^{n}-\Lambda$ and $k>0$, the Drazin inverse of $A(\bar{z})$ is given by

$$
\begin{aligned}
A(\bar{z})^{D} & =\frac{A(\bar{z})^{k} B_{t-1}(\bar{z})^{k+1}}{a_{t}(\bar{z})^{k+1}} \\
B_{t-1}(\bar{z}) & =a_{0}(\bar{z}) A(\bar{z})^{t-1}+\cdots+a_{t-2}(\bar{z}) A(s)+a_{t-1}(\bar{z}) I_{m}
\end{aligned}
$$

In the case $\bar{z} \in \mathbb{C}^{n}-\Lambda$ and $k=0$, we get $A(\bar{z})^{D}=O$.

For $\bar{z}_{i} \in \Lambda$ we can use the same algorithm again.

Proof. The proof uses the same logic as the one in [18]. For the sake of brevity the interested reader is advised to read [18].

In order to show that a multivariable polynomial matrix is zero we need the following lemma.

Lemma 6 A polynomial matrix $B\left(z_{1}, \ldots, z_{n}\right) \in \mathbb{R}\left[z_{1}, \ldots, z_{n}\right]^{m \times m}$ of degree $q_{i}$ in respect with variables $z_{i}$ is the zero polynomial matrix iff its value at $R$ distinct points is the zero matrix where

$$
R=\prod_{i=0}^{n}\left(q_{i}+1\right)
$$

In the following we suggest a computationally attractive algorithm for the calculation of Drazin inverses based on interpolation techniques and DFT.

(Evaluation of the Drazin inverse of a $n D$ polynomial matrix)

\section{Step 1}

It is easily seen from (18), that the greatest powers of the $(n+1)$ variables in $a\left(s, z_{1}, \ldots, z_{n}\right)$ are

$$
\begin{gathered}
\operatorname{deg}_{s}\left(a\left(s, z_{1}, \ldots, z_{n}\right)\right)=m:=b_{0} \\
\operatorname{deg}_{z_{1}}\left(a\left(s, z_{1}, \ldots, z_{n}\right)\right) \leq m M_{1}:=b_{1} \\
\vdots \\
\operatorname{deg}_{z_{n}}\left(a\left(s, z_{1}, \ldots, z_{n}\right)\right) \leq m M_{n}:=b_{n}
\end{gathered}
$$

So the polynomial $a\left(s, z_{1}, \ldots, z_{n}\right)$ can be written as

$$
a\left(s, z_{1}, \ldots, z_{n}\right)=\sum_{k_{0}=0}^{b_{0}} \sum_{k_{1}=0}^{b_{1}} \ldots \sum_{k_{n}=0}^{b_{n}}\left(a_{k_{0} k_{1} \ldots k_{n}}\right)\left(s^{k_{0}} z_{1}^{k_{1}} \ldots z_{n}^{k_{n}}\right)
$$


and can be numerically computed via interpolation using the following $R$ points

$$
\begin{gathered}
u_{i}\left(r_{j}\right)=W_{i}^{-r_{j}} ; i=0, \ldots, n \text { and } r_{j}=0,1, \ldots, b_{i} \\
W_{i}=e^{\frac{2 \pi j}{b_{i}+1}}
\end{gathered}
$$

where

$$
R=\prod_{i=0}^{n}\left(b_{i}+1\right)
$$

To evaluate the coefficients $a_{k_{0} k_{1} \ldots k_{n}}$ define

$$
\tilde{a}_{r_{0} r_{1} \ldots r_{n}}=\operatorname{det}\left[u_{0}\left(r_{0}\right) I_{p}-A\left(u_{1}\left(r_{1}\right), \ldots, u_{n}\left(r_{n}\right)\right)\right]
$$

From (22), (23), (24) we get

$$
\tilde{a}_{r_{0} r_{1} \ldots r_{n}}=\sum_{l_{0}=0}^{b_{0}} \sum_{l_{1}=0}^{b_{1}} \ldots \sum_{l_{n}=0}^{b_{n}}\left(a_{l_{0} l_{1} \ldots l_{n}}\right)\left(W_{0}^{-r_{0} l_{0}} \ldots W_{n}^{-r_{n} l_{n}}\right)
$$

Notice that $\left[a_{l_{0} l_{1} \ldots l_{n}}\right]$ and $\left[\tilde{a}_{r_{0} r_{1} \ldots r_{n}}\right]$ form a DFT pair and thus using the above equation and (2) we have

$$
a_{l_{0} l_{1} \ldots l_{n}}=\frac{1}{R} \sum_{r_{0}=0}^{b_{0}} \sum_{r_{1}=0}^{b_{1}} \ldots \sum_{r_{n}=0}^{b_{n}} \tilde{a}_{r_{0} r_{1} \ldots r_{n}} W_{0}^{r_{0} l_{0}} \ldots W_{n}^{r_{n} l_{n}}
$$

where $l_{i}=0, \ldots, b_{i}$.

Step 2. (Evaluate $a_{t}(\bar{z})$ as in (20))

Find $k: a_{t+1}(\bar{z})=a_{t+2}(\bar{z})=\cdots=a_{m}(\bar{z})=0$ and $a_{t}(\bar{z}) \neq 0$

Step 3. (Evaluate $r \geq t: B_{m}(\bar{z}) \equiv 0, \ldots, B_{r}(\bar{z}) \equiv 0, B_{r-1}(\bar{z}) \neq 0$ )

Consider the polynomial matrix $B_{i}(\bar{z})$. To check whether $B_{i}(\bar{z})$ is the zero matrix using lemma (6),

$$
R_{i}=\prod_{i=0}^{n}\left(i M_{i}+1\right)
$$

interpolation points are needed. In order now to determine the value of $r \geq t$ which satisfy the property : $B_{m}(\bar{z}) \equiv 0, \ldots, B_{r}(\bar{z}) \equiv 0, B_{r-1}(\bar{z}) \neq 0, R_{i}$ we use the following short algorithm

Do WHILE $\left(B_{i}(\bar{z})=0 \forall u(r)\right)$

$\mathrm{i}=\mathrm{i}-1$

Check through lemma (6) whether $B_{i}(\bar{z})=0$

END DO

$r=i$

Step 4. (Evaluation of $C(\bar{z})=A(\bar{z})^{k} B_{t-1}(\bar{z})^{k+1}$ )

The greatest powers of $z_{i}$ in

$$
C(\bar{z})=A(\bar{z})^{k} B_{t-1}(\bar{z})^{k+1}
$$

where

$$
B_{t-1}(\bar{z})=a_{0}(\bar{z}) A(\bar{z})^{t-1}+\cdots+a_{t-2}(\bar{z}) A(s)+a_{t-1}(\bar{z}) I_{m}
$$


is

$$
n_{i}=(t-1)(k+1) M_{i}
$$

Using (26), $C(\bar{z})$ can be written as

$$
C(\bar{z})=\sum_{l_{1}=0}^{n_{1}} \ldots \sum_{l_{n}=0}^{n_{n}} C_{l_{0} \cdots l_{n}}\left(z_{1}^{l_{1}} \cdots z_{n}^{l_{n}}\right)
$$

We compute $C(\bar{z})$ via interpolation using the following $R$ points

$$
\begin{gathered}
u_{i}\left(r_{j}\right)=W_{i}^{-r_{j}} ; i=1, \ldots, n \text { and } r_{j}=0,1, \ldots, n_{i} \\
W_{i}=e^{\frac{2 \pi j}{n_{i}+1}}
\end{gathered}
$$

where

$$
R=\prod_{i=1}^{n}\left\{\left(n_{i}+1\right\}\right.
$$

To evaluate the coefficients $C_{l_{0} \cdots l_{n}}$ define

$$
\tilde{C}_{r_{1} \cdots r_{n}}=C\left(u_{1}\left(r_{1}\right), \ldots u_{n}\left(r_{n}\right)\right)
$$

Using (26), (27), (29) becomes

$$
\tilde{C}_{r_{1} \cdots r_{n}}=\sum_{l_{1}=0}^{n_{1}} \ldots \sum_{l_{n}=0}^{n_{n}} C_{l_{0} \cdots l_{n}} W_{1}^{-r_{1} l_{1}} \cdots W_{n}^{-r_{n} l_{n}}
$$

which through (2)

$$
C_{l_{0} \cdots l_{n}}=\frac{1}{R} \sum_{l_{1}=0}^{n_{1}} \ldots \sum_{l_{n}=0}^{n_{n}} \tilde{C}_{r_{1} \cdots r_{n}} W_{1}^{r_{1} l_{1}} \ldots W_{n}^{r_{n} l_{n}}
$$

where $l_{i}=0, \ldots, n_{i}$.

Step 5.(Evaluation of $c(\bar{z})=a_{t}(\bar{z})^{k+1}$ )

The greatest power of $z_{i}$ appearing in $a_{t}(\bar{z})^{k+1}$ are

$$
b_{i}=t M_{i}(k+1)
$$

so $c(\bar{z})$ can be written as

$$
c(\bar{z})=\sum_{k_{1}=0}^{b_{1}} \ldots \sum_{k_{n}=0}^{b_{n}}\left(c_{k_{1} \ldots k_{n}}\right)\left(z_{1}^{k_{1}} \ldots z_{n}^{k_{n}}\right)
$$

and can be numerically computed via interpolation using the following $R$ points

$$
\begin{gathered}
u_{i}\left(r_{j}\right)=W_{i}^{-r_{j}} ; i=0, \ldots, n \text { and } r_{j}=0,1, \ldots, b_{i} \\
W_{i}=e^{\frac{2 \pi j}{b_{i}+1}}
\end{gathered}
$$


where

$$
R=\prod_{i=0}^{n}\left(b_{i}+1\right)
$$

Define

$$
\tilde{c}_{r_{1} \ldots r_{n}}=\sum_{l_{1}=0}^{b_{1}} \ldots \sum_{l_{n}=0}^{b_{n}}\left(c_{k_{1} \ldots k_{n}}\right)\left(W_{1}^{-r_{1} l_{1}} \ldots W_{n}^{-r_{n} l_{n}}\right)
$$

Using the above equation and (2) we have

$$
c_{l_{1} \ldots l_{n}}=\frac{1}{R} \sum_{r_{1}=0}^{b_{1}} \ldots \sum_{r_{n}=0}^{b_{n}} \tilde{c}_{r_{1} \ldots r_{n}} W_{1}^{r_{1} l_{1}} \ldots W_{n}^{r_{n} l_{n}}
$$

where $l_{i}=0, \ldots, b_{i}$.

Step 6. (Evaluation of the Drazin inverse)

$$
A(\bar{z})^{D}=\frac{A(\bar{z})^{k} B_{t-1}(\bar{z})^{k+1}}{a_{t}(\bar{z})^{k+1}}=\frac{C(\bar{z})}{c(\bar{z})}
$$

\section{Implementation}

In the following we present a solution to the model matching problem of multidimensional systems. Consider an open loop $2 D$ system with transfer function

$$
G(\bar{z}) \in R\left(z_{1}, z_{2}\right)^{p \times m}
$$

We want to solve the model matching problem, i.e. check whether there is an output feedback of the form

$$
u(\bar{z})=-F(\bar{z}) y(\bar{z})+v(\bar{z}), F(\bar{z}) \in \mathbb{R}(\bar{z})^{p \times m}
$$

such that the closed loop system has transfer function

$$
H(\bar{z}) \in \mathbb{R}(\bar{z})^{p \times m}
$$

and find the solution $F(\bar{z}) \in \mathbb{R}(\bar{z})^{p \times m}$ (see Figure 1).

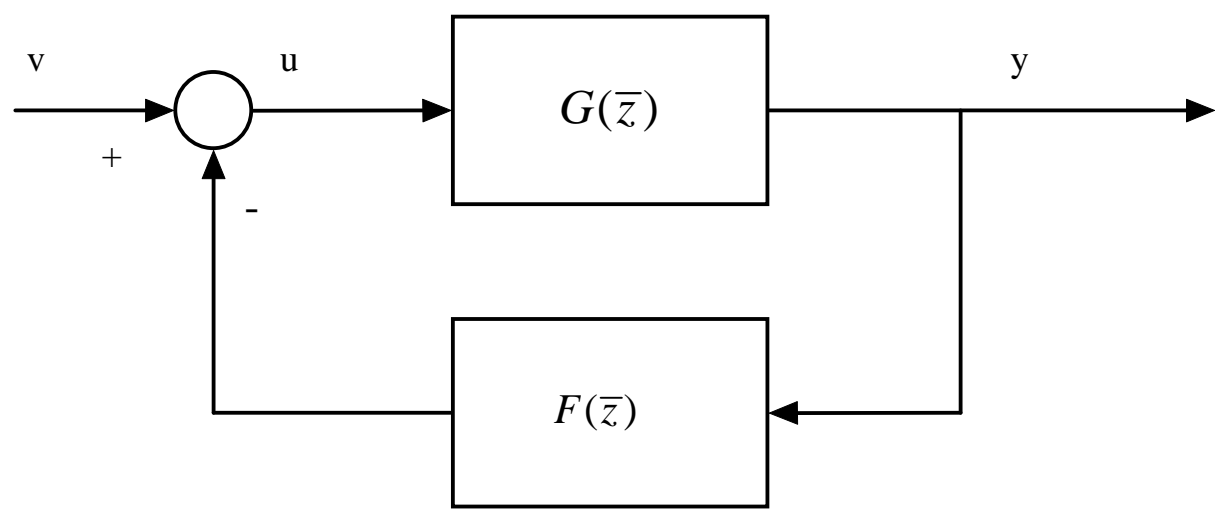

Figure 1. The model matching problem. 
Let

$$
\begin{aligned}
G(\bar{z}) & =\frac{\tilde{G}(\bar{z})}{g(\bar{z})}, \tilde{G}(\bar{z}) \in \mathbb{R}[\bar{z}]^{p \times m} \\
H(\bar{z}) & =\frac{\tilde{H}(\bar{z})}{h(\bar{z})}, \tilde{H}(\bar{z}) \in \mathbb{R}[\bar{z}]^{p \times m}
\end{aligned}
$$

where $\bar{z}=\left(z_{1}, z_{2}\right)$ and $g(\bar{z}), h(\bar{z})$ are polynomials in $\bar{z}$. Then from [13], a necessary and sufficient condition for the existence of a solution is

$$
\begin{gathered}
\tilde{G}(\bar{z}) \tilde{G}(\bar{z})^{+}[\tilde{G}(\bar{z}) h(\bar{z})-\tilde{H}(\bar{z}) g(\bar{z})] \tilde{H}(\bar{z})^{+} \tilde{H}(\bar{z}) \\
=\tilde{G}(\bar{z}) h(\bar{z})-\tilde{H}(\bar{z}) g(\bar{z})
\end{gathered}
$$

In case where the solution exists, it is given by

$$
\begin{gathered}
F(\bar{z})=\tilde{G}(\bar{z})^{+}[\tilde{G}(\bar{z}) h(\bar{z})-\tilde{H}(\bar{z}) g(\bar{z})] \tilde{H}(\bar{z})^{+}+ \\
+Y(\bar{z})-\tilde{G}(\bar{z})^{+} \tilde{G}(\bar{z}) Y(\bar{z}) \tilde{H}(\bar{z}) \tilde{H}(\bar{z})^{+}
\end{gathered}
$$

where $Y(\bar{z})$ is arbitrary to within having the dimensions of $F(\bar{z})$.

Example 7 We will solve the model matching problem as described above using as plant

$$
\begin{aligned}
G\left(z_{1}, z_{2}\right) & =\left[\begin{array}{ccc}
\frac{z_{1}-1}{z_{1}+z_{2}+1} & 0 & 0 \\
0 & \frac{z_{2}+1}{z_{1}+z_{2}+1} & 0
\end{array}\right]=\frac{1}{z_{1}+z_{2}+1}\left[\begin{array}{ccc}
z_{1}-1 & 0 & 0 \\
0 & z_{2}+1 & 0
\end{array}\right] \\
& =\frac{1}{g\left(z_{1}, z_{2}\right)} \tilde{G}\left(z_{1}, z_{2}\right)
\end{aligned}
$$

and as the desired transfer function

$$
\begin{aligned}
H\left(z_{1}, z_{2}\right) & =\left[\begin{array}{ccc}
\frac{z_{1}-1}{z_{1}-z_{2}-1} & 0 & 0 \\
0 & \frac{1}{z_{1}-z_{2}-1} & 0
\end{array}\right]=\frac{1}{z_{1}-z_{2}-1}\left[\begin{array}{ccc}
z_{1}-1 & 0 & 0 \\
0 & 1 & 0
\end{array}\right] \\
& =\frac{1}{h\left(z_{1}, z_{2}\right)} \tilde{H}\left(z_{1}, z_{2}\right)
\end{aligned}
$$

We need to compute the generalized inverse of $\tilde{G}\left(z_{1}, z_{2}\right)$, and $\tilde{H}\left(z_{1}, z_{2}\right)$. We will try and evaluate step by step $\tilde{H}\left(z_{1}, z_{2}\right)^{+}$.

Step 1.

$$
\begin{aligned}
& b_{0}=p=2 \\
& b_{1}=2 p M_{1}=2 \cdot 2 \cdot 1=4 \\
& b_{2}=2 p M_{2}=2 \cdot 2 \cdot 0=0
\end{aligned}
$$

We need $R$ interpolation points to evaluate $a\left(s, z_{1}, z_{2}\right)$ where

$$
R=\prod_{i=0}^{2}\left(b_{i}+1\right)=(2+1)(4+1)(0+1)=15
$$


Those points (in $3 D$ space) are $\left(u_{0}\left(r_{0}\right), u_{1}\left(r_{1}\right), u_{2}\left(r_{2}\right)\right)$ where

$$
\begin{aligned}
u_{i}\left(r_{j}\right) & =W_{i}^{-r_{j}}, i=0,1,2 ; r_{j}=0, \ldots, b_{i} \\
W_{i} & =e^{\frac{2 \pi j}{b_{i}+1}}
\end{aligned}
$$

and after some calculations

$$
\begin{aligned}
& u_{0}(0)=1, u_{0}(1)=e^{-\frac{2 \pi j}{3}}, u_{0}(2)=e^{-\frac{4 \pi j}{3}} \\
& u_{1}(0)=1, u_{1}(1)=e^{-\frac{2 \pi j}{5}}, u_{1}(2)=e^{-\frac{4 \pi j}{5}}, u_{1}(3)=e^{-\frac{6 \pi j}{5}}, u_{1}(4)=e^{-\frac{8 \pi j}{5}} \\
& u_{2}(0)=1
\end{aligned}
$$

Now evaluating (11) at the above points, we have

$\left[\tilde{a}_{i j 0}\right]=\left[\begin{array}{ccccc}0 & 0 & 0 & 0 & 0 \\ 1.73 j & -1.77+3.33 j & 2.54+7.45 j & 6.23+1.07 j & 0.49-0.60 j \\ -1.73 j & 0.49+0.609 j & 6.23-1.07 j & 2.54-7.45 j & -1.77-3.33 j\end{array}\right]$

where in order to save space we wrote $\tilde{a}_{i j 0}$ in a table form. Now using the inverse Fourier transform we finally get after elimination of the entries smaller than $10^{-10}$ that

$$
\left[a_{i j 0}\right]=\left[\begin{array}{ccccc}
1 & -2 & 1 & 0 & 0 \\
-2 & 2 & -1 & 0 & 0 \\
1 & 0 & 0 & 0 & 0
\end{array}\right]
$$

i.e.

$$
a\left(s, z_{1}, z_{2}\right)=s^{2}-z_{1}^{2} s+2 z_{1} s-2 s+z_{1}^{2}+1
$$

Step 2.

$$
a\left(s, z_{1}, z_{2}\right)=s^{2}+\left(-z_{1}^{2}+2 z_{1}-2\right) s+\left(z_{1}^{2}-2 z_{1}+1\right)
$$

so

$$
k=2
$$

and

$$
a_{2}\left(z_{1}, z_{2}\right)=z_{1}^{2}-2 z_{1}+1
$$

Step 3.

$$
\begin{aligned}
& n_{1}=3 \\
& n_{2}=0
\end{aligned}
$$

and the $2 D$ interpolation points needed are $R=4$

$$
\begin{aligned}
& u_{1}(0)=1 ; u_{1}(1)=e^{-\frac{\pi j}{2}} ; u_{1}(2)=e^{-\pi j} ; u_{1}(3)=e^{-\frac{3 \pi j}{2}} \\
& u_{2}(0)=1
\end{aligned}
$$


We then evaluate the matrix (14) at the above points resulting in the next sequence of 4 matrices

$$
\underbrace{\left[\begin{array}{ll}
0 & 0 \\
0 & 0 \\
0 & 0
\end{array}\right]}_{\tilde{C}_{00}}, \underbrace{\left[\begin{array}{cc}
1+j & 0 \\
0 & -2 j \\
0 & 0
\end{array}\right]}_{\tilde{C}_{01}}, \underbrace{\left[\begin{array}{cc}
2 & 0 \\
0 & -4 \\
0 & 0
\end{array}\right]}_{\tilde{C}_{02}}, \underbrace{\left[\begin{array}{cc}
1-j & 0 \\
0 & 2 j \\
0 & 0
\end{array}\right]}_{\tilde{C}_{03}}
$$

which by inverse Fourier transform returns the coefficients of $C(\bar{z})$ as in (13)

$$
C\left(z_{1}, z_{2}\right)=\left[\begin{array}{cc}
1-z_{1} & 0 \\
0 & 1 \\
0 & 0
\end{array}\right]
$$

Step 4.

The generalized inverse is

$$
H\left(z_{1}, z_{2}\right)^{+}=-\frac{1}{a_{k}\left(z_{1}, z_{2}\right)} C\left(z_{1}, z_{2}\right)=\left[\begin{array}{cc}
-\frac{1}{z_{1}^{2}-2 z_{1}-1} & 0 \\
0 & 1 \\
0 & 0
\end{array}\right]
$$

Using the same algorithm we can evaluate $G\left(z_{1}, z_{2}\right)^{+}$

$$
G\left(z_{1}, z_{2}\right)^{+}=\left[\begin{array}{cc}
\frac{z_{1}+z_{2}+1}{z_{1}-1} & 0 \\
0 & \frac{z_{1}+z_{2}+1}{z_{2}+1} \\
0 & 0
\end{array}\right]
$$

and afterwords check whether (32) holds, which is indeed true. So the general solution to the model matching problem is the result of (33)

$$
F\left(z_{1}, z_{2}\right)=\left[\begin{array}{cc}
\frac{2\left(z_{2}+1\right)}{z_{1}-1} & 0 \\
0 & \frac{-z_{2}^{2}+z_{1} z_{2}-3 z_{2}-2}{z_{2}+1} \\
y_{31}\left(z_{1}, z_{2}\right) & y_{32}\left(z_{1}, z_{2}\right)
\end{array}\right]
$$

where $y_{31}\left(z_{1}, z_{2}\right)$ and $y_{32}\left(z_{1}, z_{2}\right)$ are arbitrary real rational functions.

\section{Conclusions}

In this paper two algorithms have been presented for determining the generalized and Drazin inverse of $n D$ polynomial matrices. The algorithms are based on the discrete Fourier transform and therefore have the main advantages of speed and robustness in contrast to other known algorithms. The theoretical work is accompanied by an example that tackles the problem of model matching. Other applications of the theory introduced, include the solution of multivariable Diophantine equations and its application to control system synthesis problems, the computation of the transfer function matrix of multidimensional systems, 
the solution of multidimensional AutoRegressive representations etc. The above mentioned algorithms may be easily extended in order to determine other kind of inverses such as $\{2\},\{1,2\},\{1,2,3\}$ and $\{1,2,4\}$ inverses of multivariable polynomial matrices by using the Leverrier-Faddeev algorithms presented in $[19]$.

\section{References}

[1] Antoniou, G.: 2001, 'Transfer function computation for generalized ndimensional systems'. Journal of the Franklin Institute 338, 83-90.

[2] Antoniou, G. E., G. O. A. Glentis, S. J. Varoufakis, and D. A. Karras: 1989, 'Transfer function determination of singular systems using the DFT'. IEEE Trans. Circuits and Systems 36(8), 1140-1142.

[3] Decell, Jr., H. P.: 1965, 'An application of the Cayley-Hamilton theorem to generalized matrix inversion'. SIAM Rev. 7, 526-528. (extended in [20]).

[4] Drazin, M. P.: 1958, 'Pseudo inverses in associative rings and semigroups'. Amer. Math. Monthly 65, 506-514.

[5] Dudgeon, D. and R. Mersereau: 1984, Multidimensional Digital Signal Processing. Prentice Hall.

[6] Frigo, M. and S. Johnson: 1998, 'FFTW: An Adaptive Software Architecture for the FFT'. In: ICASSP conference proceedings (vol. 3, pp. 13811384).

[7] Greville, T. N. E.: 1973, 'The Souriau-Frame algorithm and the Drazin pseudoinverse'. Linear Algebra and Appl. 6, 205-208.

[8] Güyer, T., O. Kı ymaz, G. Bilgici, and Ş. Mirasyedioğlu: 2001, 'A new method for computing the solutions of differential equation systems using generalized inverse via Maple'. Appl. Math. Comput. 121(2-3), 291-299.

[9] Hromcik, M. and M. Sebek: 2001, 'Fast Fourier transform and linear polynomial matrix equations'. In: Proceedings of the 1rst IFAC Workshop on Systems Structure and Control, Prague, Czech Republic.

[10] Jones, J., N. P. Karampetakis, and A. C. Pugh: 1998, 'The computation and application of the generalized inverse via Maple'. J. Symbolic Comput. 25(1), 99-124.

[11] Karampetakis, N. and P. Stanimirovic: 2001, 'On the computation of the Drazin inverse of a polynomial matrix'. In: Proceedings of the 1rst IFAC Symposium on Systems Structure and Control, Prague, Czech Republic.

[12] Karampetakis, N. P.: 1997a, 'Computation of the generalized inverse of a polynomial matrix and applications'. Linear Algebra Appl. 252, 35-60. 
[13] Karampetakis, N. P.: 1997b, 'Generalized inverses of two-variable polynomial matrices and applications'. Circuits Systems Signal Process. 16(4), 439-453.

[14] Karampetakis, N. P. and P. Tzekis: 2001, 'On the computation of the generalized inverse of a polynomial matrix'. IMA J. Math. Control Inform. 18(1), 83-97.

[15] Paccagnella, L. E. and G. L. Pierobon: 1976, 'FFT calculation of a determinantal polynomial'. IEEE Trans. on Automatic Control pp. 401-402.

[16] Penrose, R.: 1955, 'A Generalized Inverse for Matrices'. Proceedings of the Cambridge Philosophical Society 51, 406-413.

[17] Schuster, A. and P. Hippe: 1992, 'Inversion of polynomial matrices by interpolation'. IEEE Trans. Automat. Control 37(3), 363-365.

[18] Stanimirovic, P. and N. Karampetakis: 2000, 'Symbolic implementation of Leverrier-Faddeev algorithm and applications.'. In: Proceedings of the 8th IEEE Medit. Conference on Control and Automation, Patra, Greece.

[19] Stanimirovic, P. S. and M. B. Tasic: 2002, 'A problem in computation of pseudoinverses'. To appear in Applied Mathematics and Computation.

[20] Wang, G.: 1987, 'A finite algorithm for computing the weighted MoorePenrose inverse $A_{M N}^{+}$'. Appl. Math. Comput. 23(4), 277-289. 